ARTICLE

DOI: $10.1038 / \mathrm{s} 41467-017-02312-7$

\title{
Trapping IgE in a closed conformation by mimicking CD23 binding prevents and disrupts FceRI interaction
}

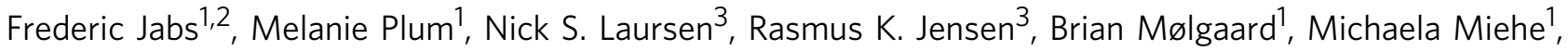
Marco Mandolesi (1) ${ }^{1}$, Michèle M. Rauber ${ }^{4,5}$, Wolfgang Pfützner ${ }^{4}$, Thilo Jakob ${ }^{5}$, Christian Möbs (D) ${ }^{4}$, Gregers R. Andersen ${ }^{3} \&$ Edzard Spillner (i) ${ }^{1}$

Anti-lgE therapeutics interfere with the ability of $\lg E$ to bind to its receptors on effector cells. Here we report the crystal structure of an anti-lgE single-domain antibody in complex with an IgE Fc fragment, revealing how the antibody inhibits interactions between IgE and the two receptors FceRI and CD23. The epitope overlaps only slightly with the FceRI-binding site but significantly with the CD23-binding site. Solution scattering studies of the IgE Fc reveal that antibody binding induces a half-bent conformation in between the well-known bent and extended IgE Fc conformations. The antibody acts as functional homolog of CD23 and induces a closed conformation of IgE Fc incompatible with FcERI binding. Notably the antibody displaces IgE from both CD23 and FceRI, and abrogates allergen-mediated basophil activation and facilitated allergen binding. The inhibitory mechanism might facilitate strategies for the future development of anti-lgE therapeutics for treatment of allergic diseases.

\footnotetext{
${ }^{1}$ Immunological Engineering, Department of Engineering, Aarhus University, 8000 Aarhus, Denmark. ${ }^{2}$ Institute of Organic Chemistry, Department of Chemistry, University of Hamburg, 20146 Hamburg, Germany. ${ }^{3}$ Department of Molecular Biology and Genetics, Aarhus University, 8000 Aarhus, Denmark. ${ }^{4}$ Clinical \& Experimental Allergology, Department of Dermatology and Allergology, Philipps University Marburg, 35043 Marburg, Germany. ${ }^{5}$ Department of Dermatology and Allergology University Medical Center Giessen and Marburg, Justus-Liebig University Giessen, 35385 Giessen, Germany. Frederic Jabs, Melanie Plum, Nick Stub Laursen and Rasmus K. Jensen contributed equally to this work. Christian Möbs, Gregers R. Andersen and Edzard Spillner jointly supervised this work. Correspondence and requests for materials should be addressed to N.S.L. (email: nsl@mbg.au.dk)

or to E.S. (email: e.spillner@eng.au.dk)
} 
$\Lambda$ llergic diseases can be linked to $\operatorname{IgE}$ antibodies present in the circulation and on the surface of a variety of cell types ${ }^{1}$. Although the least abundant type of antibodies, IgE exhibits a variety of structural peculiarities with major functional consequences. IgE acts as a key molecule in a network of proteins, including the high-affinity IgE receptor FceRI, the low-affinity receptor $\mathrm{CD} 23$, and galectins, e.g., galectin- $3^{2}$. Upon crosslinking by allergens, IgE bound to FceRI on mast cells and basophils triggers degranulation, release of proinflammatory mediators, and immediate reactions ${ }^{2}$.

$\operatorname{IgE}$ is an evolutionarily conserved and heavily glycosylated heterotetramer (Fig. 1a) with the epsilon heavy chain having four constant domains. The IgE Fc binds to the human FceRI complex that is expressed as an $\alpha \beta \gamma 2$ tetramer or an $\alpha \gamma 2$ trimer lacking the signal amplifying $\beta$-subunit ${ }^{3-5}$. The $\alpha$-chain of the FceRI displays an affinity for IgE in the range of $10^{11} \mathrm{M}^{-1}$, providing the basis for long-term stability on effector cells and half-life of $\sim 10$ days ${ }^{6}$.

Strategies to reduce increased levels of IgE and to limit effector cell degranulation included the development of antagonistic anti$\mathrm{IgE}$ antibodies and antibody alternatives including a DARPin and aptamers ${ }^{7}$. The only approved anti-IgE antibody, omalizumab, primarily prevents interaction of free IgE with its receptor on effector cells $s^{8-10}$ and eventually reverses phenotypic and functional effects of IgE such as enhanced FceRI levels on effector cells $^{11,12}$. Not all patients with allergic asthma benefit from treatment ${ }^{13}$ and failure may also be caused by pharmacologically active IgE:omalizumab complexes ${ }^{14}$ that hamper correct dosing of anti-IgE ${ }^{15}$. Second-generation anti-IgE molecules such as ligelizumab and MEDI4212 are currently under investigation, but initial results suggest limited improvement. Basic structural and functional aspects of anti-IgE, e.g., the mechanism of rapid improvement in chronic urticaria, remain unclear ${ }^{16,17}$.

Key for receptor binding and therefore anti-IgE concepts is the IgE Fc that may adopt strongly bent or extended structures with most striking differences in the positioning of the CE2 domains ${ }^{18,19}$. Furthermore, the C $\varepsilon 3-4$ sub-fragment adopts different conformational states ranging from closed to open depending on the spacing of the $\mathrm{C} \varepsilon 3$ domains and their distance to the C 84 domains ${ }^{20}$. This conformational flexibility allows the CE3 domains to rotate (swing) closer to or farther away from each other (Fig. 1b, c).

Structural studies have unraveled how IgE interacts in a highly ordered and specific manner with its receptors. The FceRIbinding site on the IgE Fc is located primarily on the Ce3 domain whereas $\mathrm{CD} 23$ binds to a site involving both the $\mathrm{C} \varepsilon 3$ and $\mathrm{C} \varepsilon 4$ domains (Fig. 1a) ${ }^{21,22}$. FceRI binds to the open and CD23 to the closed conformation of $\mathrm{C} \varepsilon 3-4$ domains (Fig. 1b) making binding of the two receptors mutually exclusive and preventing overlap of

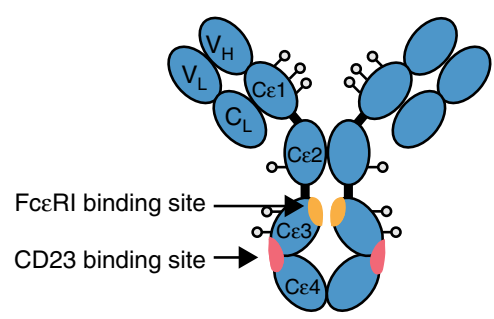

b

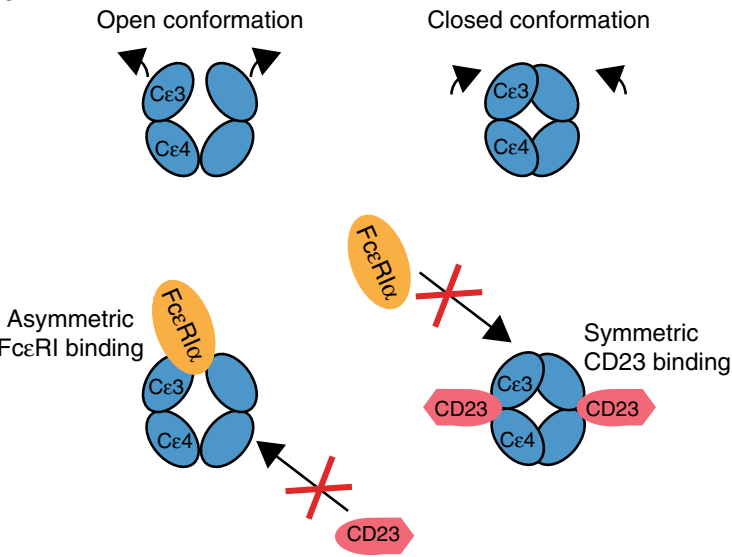

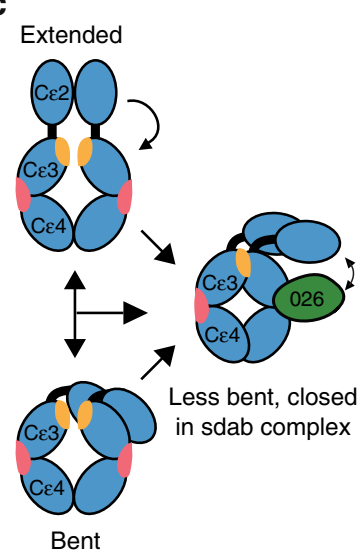

d

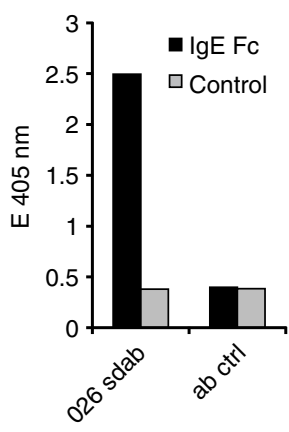

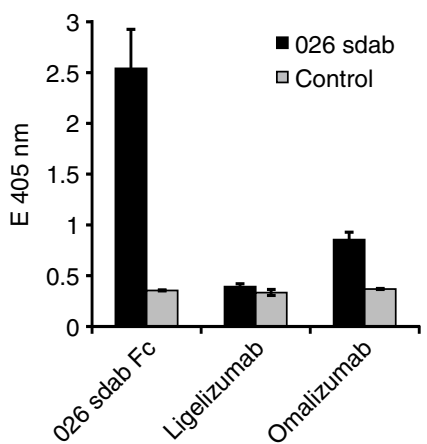

f

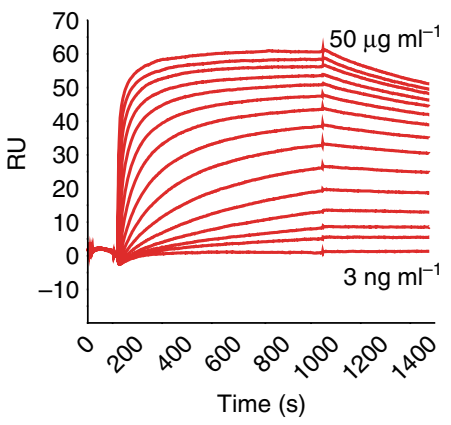

g

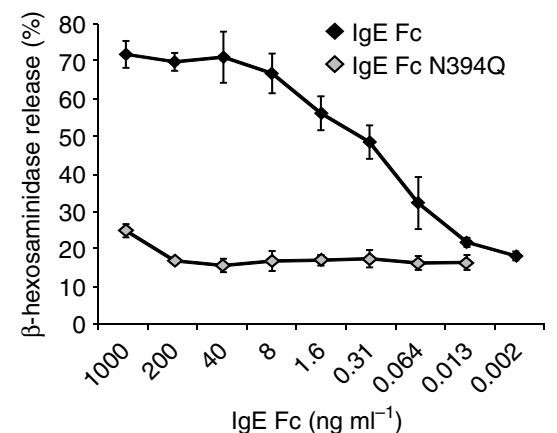

Fig. 1 Organization and conformational rearrangements of the IgE Fc. a lgE and the binding sites of the FceRI (orange) and CD23 (pink) (adapted from ref. ${ }^{23}$ ). The glycosylations are indicated by dots. b Representation of the open and closed conformations of the IgE Fc C $83-4$ domains, and the mutual allosteric inhibition by FceRl $\alpha$ (orange) and CD23 (pink). c Representation of the bent and extended conformation of IgE Fc C $\varepsilon 2-4$ and the conformation in the 026 sdab complex, together with the relative position of the $C \varepsilon 2$ domains. $\mathbf{d}$ Immunoreactivity of the 026 sdab to recombinant IgE Fc was assessed by ELISA. e Interference of the 026 sdab with different anti-lgE antibodies was assessed by sandwich ELISA using 026 sdab for capturing IgE Fc. Data are mean \pm s.d. Detection of bound anti-lgE antibodies was performed using anti-lgG antibodies coupled to alkaline phosphatase. $\mathbf{f}$ The affinity of the 026 antiIgE binding to immobilized IgE Fc was assessed by surface plasmon resonance. $\mathbf{g}$ Biological activity of recombinant IgE Fc and an lgE Fc lacking the glycan at N394 in mediator-release assays. Data are mean \pm s.d. RBL-SX38 cells expressing the human FcERI were sensitized with IgE Fc. Degranulation was induced by the addition of anti-lgE and monitored by released $\beta$-hexosaminidase activity 


\begin{tabular}{|c|c|}
\hline \multicolumn{2}{|l|}{ Data collection } \\
\hline Data set & IgE-Fc-026 \\
\hline X-ray source & Diamond 124 \\
\hline Space group & $P 4_{1} 2_{1} 2$ \\
\hline Unit cell parameters & $\begin{array}{l}a=102.0, b=102.0, c=300.4 \AA \\
\alpha=\beta=\gamma=90^{\circ}\end{array}$ \\
\hline Resolution $(\AA)^{a}$ & $19.96-3.40(3.52-3.40)$ \\
\hline Unique reflections $^{a}$ & $22,564(2190)$ \\
\hline Multiplicity $^{a}$ & $12.3(10.5)$ \\
\hline $\mathrm{I} / \sigma(I)^{\mathrm{a}}$ & $8.3(1.11)$ \\
\hline Completeness $^{a}$ & $0.99(1.00)$ \\
\hline$R_{\text {meas }}{ }^{a}$ & $0.35(2.37)$ \\
\hline$R_{\text {pim }}{ }^{a}$ & $0.10(0.71)$ \\
\hline$R_{\text {merge }}{ }^{a}$ & $0.34(2.25)$ \\
\hline $\mathrm{CC}_{1 / 2} \mathrm{a}^{\mathrm{a}}$ & $0.99(0.76)$ \\
\hline \multicolumn{2}{|l|}{ Refinement statistics } \\
\hline Reflections in refinement & 22,560 \\
\hline$R_{\text {work }}$ & 0.2123 \\
\hline$R_{\text {free }}$ & 0.2408 \\
\hline Average $B$-value $\left(\AA^{2}\right)$ & 111.4 \\
\hline Macromolecule $\left(\AA^{2}\right)$ & 111.4 \\
\hline Carbohydrates $\left(\AA^{2}\right)$ & 121.7 \\
\hline Wilson $B$-value $\left(\AA^{2}\right)$ & 108.2 \\
\hline Protein atoms & 5226 \\
\hline Carbohydrate atoms & 166 \\
\hline \multicolumn{2}{|l|}{ Ramachandran statistics (\%) } \\
\hline Favored & 97.12 \\
\hline Allowed & 2.88 \\
\hline Outliers & 0.00 \\
\hline \multicolumn{2}{|l|}{ RMSD from ideal geometry } \\
\hline Bond angles $\left({ }^{\circ}\right)$ & 0.86 \\
\hline Bond length $(\AA)$ & 0.004 \\
\hline Clashscore & 3.76 \\
\hline PDB ID & 5NQW \\
\hline
\end{tabular}

a Values in parentheses are for highest-resolution shell. Statistics were calculated by XSCALE (scaling), phenix.refine (refinement), and MOLPROBITY (validation)

the two pathways. Generally, anti-IgE antibodies are supposed to bind in proximity to the FceRI-binding site in order to interfere with the receptor binding. A recent study of the omalizumab:IgE complex defined steric interference with FceRI as the main mechanism for its inhibitory effects ${ }^{23}$.

Single-domain antibodies (sdabs) are the antigen-binding moiety of heavy chain antibodies occurring in camelid species and cartilaginous fishes ${ }^{24,25}$. Their small size and peculiar biochemical features render sdabs highly versatile molecules. Here we report the crystal structure and structure in solution of the human IgE Fc in complex with the sdab 026, a llama-derived, humanized sdab that was selected against IgE. Our data show that each $\operatorname{IgE~Fc~is~targeted~by~two~sdab~having~an~epitope~largely~}$ distinct from the FceRI binding site but overlapping significantly with the CD23-binding site. Inhibition of IgE binding to FceRI occurs through an allosteric mechanism where the IgE Fc is trapped in a closed, less bent conformation similar to the CD23bound conformation. Hence, our data provide evidence for a mechanism of inhibition with major implications for the biology and therapeutic targeting of IgE.

\section{Results}

Structure of IgE Fc bound by two single-domain antibodies. The $026 \mathrm{sdab}$, recently developed for IgE targeting in allergic diseases ${ }^{26}$, was produced in bacteria and purified from supernatant in yields of up to $80 \mathrm{mg} \mathrm{l}^{-1}$. The IgE Fc Ce2-4 was purified from the supernatant of mammalian cell culture (Supplementary Figure 1a). The proteins exhibited immunoreactivity in ELISA (Fig. 1d). Sandwich ELISA analysis using immobilized 026 sdab as capturing antibody suggested the presence of two $026 \mathrm{sdab}$ binding sites in the IgE Fc as bound IgE could be detected using a bivalent 026 sdab Fc fusion molecule (Fig. 1e). Residual binding of omalizumab to 026 sdab bound IgE Fc suggests a partial overlap of the 026 sdab epitope with the omalizumab epitope. In contrast, the lack of ligelizumab binding might indicate either significant overlap of the epitopes or conformational changes incompatible with ligelizumab binding (Fig. 1e). Surface plasmon resonance analysis using IgE Fc coupled to the sensor surface revealed a dissociation constant $K_{\mathrm{D}}=1.4 \mathrm{nM}$ for the sdab, slightly higher than that reported for binding to IgE (Fig. 1f ${ }^{26}$. Mediator release assays verified biological activity of the IgE Fc and relevance of the glycan at N394 (Fig. 1g).

After purification of the IgE Fc:026 sdab complex (Supplementary Figure 1), we obtained crystals that diffracted X-rays to a maximum resolution of $3.4 \AA$ (Table 1) and determined the structure by molecular replacement. The asymmetric unit contains two copies of the 026 sdab and one IgE Fc molecule. During the process of structure determination it became clear that the crystals contained the IgE Fc Ce3-4 fragment and not the IgE Fc Ce2-4 as expected. Apparently a small fraction of IgE Fc CE3-4 was sufficient for crystallization of the complex between the sdab and this $\mathrm{Fc}$ fragment. Iterative rebuilding and refinement resulted in a final structure with $R_{\text {work }} / R_{\text {free }}$ values of $0.2123 /$ 0.2408 (Table 1). Most residues could be traced with confidence and clear electron density is present for the majority of residues at the sdab:Fc interface (Supplementary Figure 2). The 026 sdab binds symmetrically to the IgE Ce3-4 forming a 2:1 complex where each sdab:Fc interaction buries $\sim 800 \AA^{2}$ upon complex formation (Fig. 2a, b). The contacts involve surface areas that are clearly different from the FceRI $\alpha$ binding loops (Fig. 2b, $\mathrm{c}$ and Supplementary Figure 3a); thus, the 026 sdab does not inhibit IgE: FceRI interactions by blocking the binding site. Instead, the sdab is positioned between $\mathrm{C} \varepsilon 3$ and $\mathrm{C} \varepsilon 4$ from two different $\varepsilon$-chains, a region also containing the binding site of $\mathrm{CD} 23^{22}$

As expected the complementarity determining regions (CDRs) in the sdab are responsible for the majority of the $\operatorname{IgE}$ contacts. The sdab bridges the C $\varepsilon 3$ and $\mathrm{C} \varepsilon 4$ domains from each $\varepsilon$-chain in the IgE Fc dimer (Fig. 2a, b) with the C 83 and the C 84 domains contributing with $30 \%$ and $70 \%$, respectively, of the total buried surface area on IgE Fc. Although the resolution of the diffraction data is limited to $3.4 \AA$, the quality of the electron density map at the sdab:IgE Fc interface allowed us to suggest putative intermolecular hydrogen bonds and salt bridges. The CDR3 is inserted between the C 83 and $C \varepsilon 4$ domains and contacts several residues in the D-E loop of Ce4. Residues D99 and D110 in the sdab possibly engage in electrostatic interactions with the side chain of IgE K497, and E108 appears to make hydrogen bonds to IgE Fc residues 498-501 (Fig. 2c). Several residues in sdab CDR1 and CDR3 may recognize R393 in the Fc C-D loop through hydrogen bonds and salt bridges (Fig. 2d). In addition to the contacts mediated by the CDRs, residues in framework region 2 (FR2) also contribute. A glutamine (Q39) is likely to engage in two hydrogen bonds with the main chain of IgE R440 in the linker between Ce3 and Ce4, and sdab R44 is in position for forming hydrogen bonds to A442 and N468 (Fig. 2e).

In order to validate the crystal structure we mutated residues within sdab CDRs observed to contact IgE (Supplementary Figure $4 \mathrm{a}-\mathrm{c})$. Most mutations led to a significant reduction of sdab binding to IgE Fc (Supplementary Figure 4d). In particular, mutation of residues E108, D110 and Y112 in CDR3 abolished IgE binding suggesting a critical role in the interaction and 
a

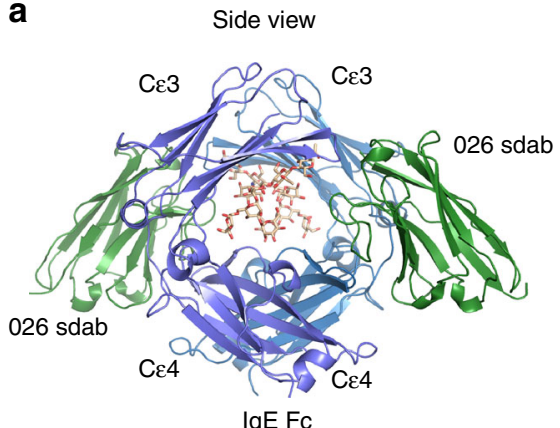

b

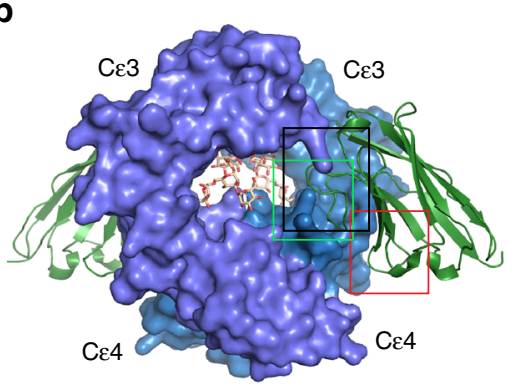

Bottom view
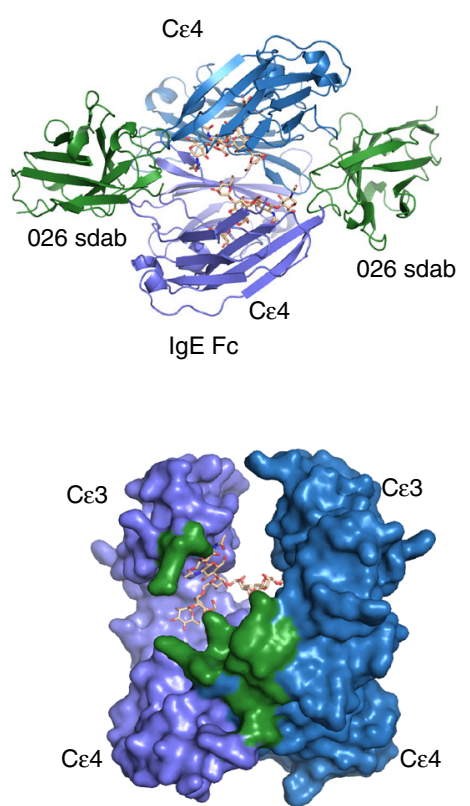

C

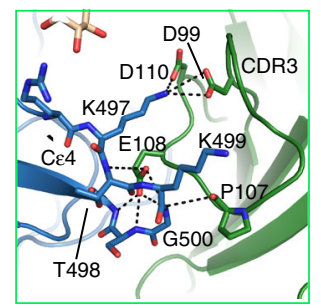

d

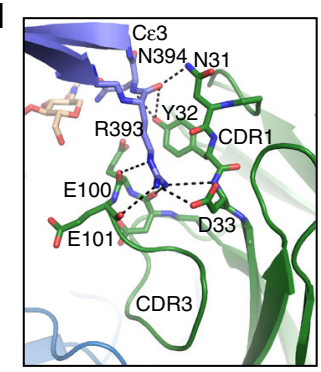

e

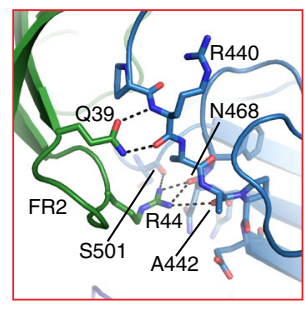

Fig. 2 The 026:lgE Fc complex and the sdab epitope. a A cartoon representation showing IgE Fc C $83-4$ and two 026 sdab molecules binding symmetric sites (green, 026; blue, IgE Fc heavy chain 1; violet, IgE Fc heavy chain 2). The side view of the complex shows the 026 sdab approaching perpendicularly

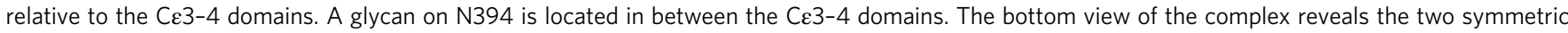
026 epitopes on the $C_{\varepsilon} 3$ and $C_{\varepsilon} 4$ domains. b Side view of $\operatorname{lgE}$ with bound 026 sdab (left) and without (right). IgE residues in contact with 026 sdab are colored green. c-e Detailed view of the IgE Fc:026 sdab interface, with putative hydrogen bonds and salt bridges shown as dotted lines

supporting that the contribution of CDR3 to the interaction is essential.

In IgE, N394 carries an oligomannosidic glycan that is essential for biological activity ${ }^{27}$. In our structure, we observe this as a $(N$ acetylglucosamine)2(mannose)5 (NAG2MAN5) hepta-saccharide, which represents the major form in natural IgE derived from myeloma cells as well as allergic patients (Supplementary Figure 5$)^{28}$. The hepta-saccharide has been reported for the structure of free IgE Fc and in both structures the glycans contact the same residues suggesting limited flexibility of the glycan ${ }^{29}$. Despite proximity of the sdab epitope to the glycan there is no direct contact, but water-mediated contacts not visible at this resolution may be present.

Structural basis for the inhibitory activity of the sdab. Recently, structures of the Fab fragments of omalizumab ${ }^{23}$, two other antiIgE antibodies ${ }^{18,30}$, and a DARPin ${ }^{31}$ in complex with IgE Fc domains have been described. All these molecules preferentially bind to the Ce3 domain of IgE critically involved in FceRI $\alpha$ binding (Supplementary Figure 3). The sdab in contrast exhibits a binding mode clearly different from the DARPin and the Fab fragments, and the IgE residues interacting with sdab CDRs are also largely distinct from those that engage FceRI $\alpha$. Hence, direct competition between the sdab and FceRI for binding must be negligible (Supplementary Figure 3a). In contrast, there is a significant overlap between the sdab and the CD23 epitopes (Fig. 3a, b). In particular, IgE Fc residues $\mathrm{S} 437-\mathrm{R} 440$ interact with the sdab and also provide an important part of the CD23 epitope (Fig. 3c).

Binding to FceRI and CD23 stabilizes different conformations of the IgE Ce3-4 domains, the open and closed state, respectively. A direct comparison shows that within the sdab complex IgE Fc adopts a closed conformation similar to that adopted upon CD23 binding, which is incompatible with binding to FceRI (Fig. 3d and Supplementary Figure 6). The sdab-mediated stabilization of the closed conformation provides a rationale for the sdab's inhibitory mechanism with respect to IgE/FceRI interaction, whereas CD23 binding to $\operatorname{IgE}$ is prevented by steric hindrance.

Effect of single-domain antibody on FceRI and CD23 binding. From the structural data it is evident that the binding sites of FceRI and the sdab, which was developed as anti-IgE blocking IgE binding to FceRI, are without significant overlap that could explain an inhibitory activity of the sdab by competition. As expected, the anti-IgE sdab prevented binding of IgE and release 
a

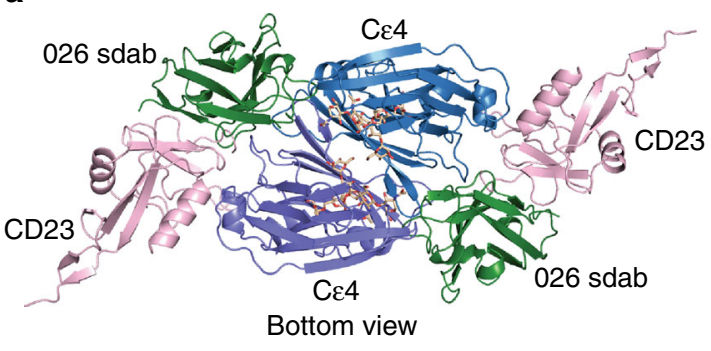

b

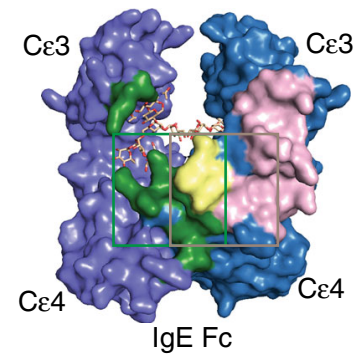

C

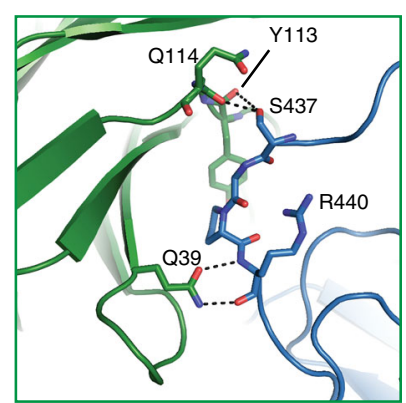

d

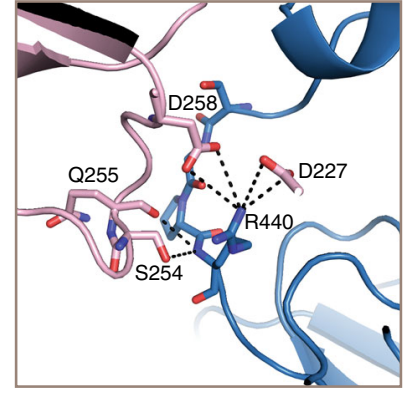

Open conformation

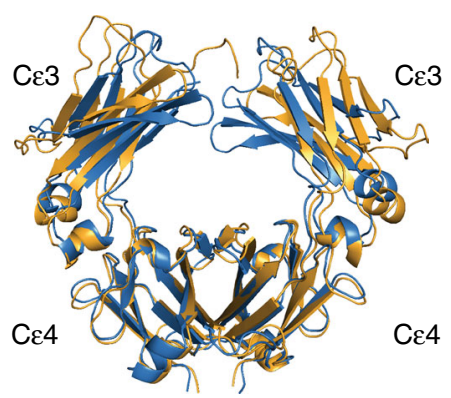

IgE Fc (026 sdab) IgE FC (FceRl $\alpha)$

Fig. 3 Comparison of sdab 026 and CD23 epitopes and IgE Fc conformation. a An overlay of the IgE Fc:026 sdab complex with the IgE Fc:CD23 complex shows the proximity of the epitopes of the sdab and CD23. b Surface representation of the IgE Fc with the 026 sdab epitope displayed in green, the CD23 epitope in pink and shared epitope residues in yellow. c Detailed view of the interface between 026 (left) and CD23 (right, PDB ID: 4GKO) and IgE residues 437-440. Putative hydrogen bonds or salt bridges are shown as dotted black lines. $\mathbf{d}$ Superposition of the IgE Fc domains reveals the similarity of the IgE Ce3-4 closed conformations in the complex with the sdab in blue and CD23 in pink (left) in contrast to the open conformation in the complex with FceRI in yellow (right, PDB ID: 1F6A)

of mediators from RBL-SX38 effector cells (Fig. 4a). Recently an anti-IgE DARPin (and omalizumab at higher concentrations) was shown to accelerate dissociation of IgE from FceRI ${ }^{23,31}$. Therefore we assessed the effect of the sdab on IgE bound to the FceRI.

Flow cytometry analysis of surface IgE on human basophils obtained from three allergic patients sensitized to inhalative and injected allergen showed that treatment with the 026 sdab for $15 \mathrm{~min}$ reduced the amount of surface $\operatorname{IgE}$ to $\sim 30 \%$ (Fig. $4 \mathrm{~b}$, Supplementary Table 1). Prolonging the incubation time further reduced surface IgE to $\sim 20 \%$. Neither the inactive sdab mutant 112 nor omalizumab exhibited comparable reduction. Incubation of immobilized IgE:FceRI complexes with 026 sdab and omalizumab supported the displacement of $\operatorname{IgE}$ by the $026 \mathrm{sdab}$ (Supplementary Figure 7).

Next, we analyzed the impact on basophil activation for a panel of six patients with birch pollen allergy (Supplementary Table 1). The high prevalence of birch pollen allergy and the fact that birch pollen major allergen Bet $\mathrm{v} 1$ is the predominant allergen for the vast majority of birch pollen allergic patients in Northern Europe allows for a good comparability of obtained results. Therefore we analyzed changes in basophil allergen threshold sensitivity, CDsens, to Bet $\mathrm{v} 1$ in basophil activation tests. CDsens is a well-established measure to quantify effector cell sensitivity to an allergen and reflects the amount of allergen needed for efficient activation. In accordance with the reduced surface IgE, the 026 sdab significantly reduced CDsens for patient's basophils by 50-95\%. (Fig. 4c-e). Levels of specific IgE (sIgE) to Bet v 1 and total $\operatorname{IgE}(\mathrm{tIgE})$ in serum suggest that lower concentrations of $\operatorname{sigE}$ and as a consequence thereof smaller ratios of $\operatorname{sgE}$ to $\operatorname{tgEE}$ translate into a more efficient reduction of CDsens (Supplementary Figure 8). These findings are in line with the observation that polysensitization can be accompanied with a less severe phenotype of the allergic response.

To validate the observed overlap between the sdab and CD23 epitopes, we next assessed the capability of the sdab for interfering with CD23 binding of IgE. ELIFAB assays are able to detect the binding of preformed oligovalent IgE:allergen complexes to $\mathrm{CD} 23^{32}$. The monovalent sdab has a significantly higher affinity to $\operatorname{IgE}$ as compared to monovalent CD23 $\left(K_{\mathrm{d}}: 2 \mu \mathrm{M}\right)$. Sera of patients with elevated level of specific IgE 
a
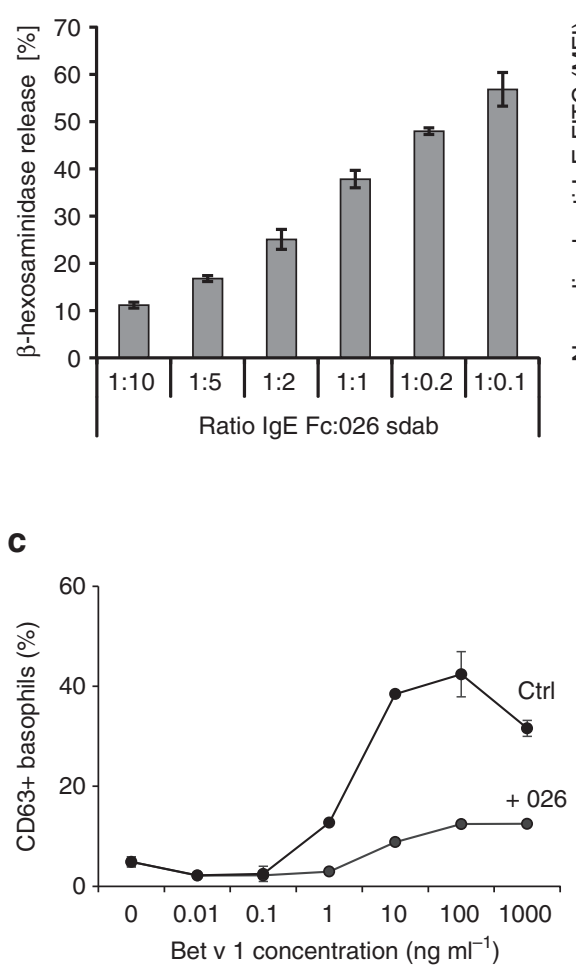

b

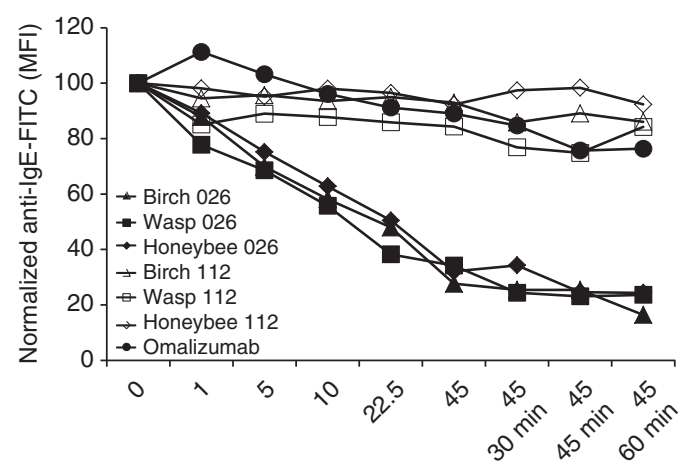

sdab concentration $\left(\mu \mathrm{mol} \mathrm{I}{ }^{-1}\right)$

d
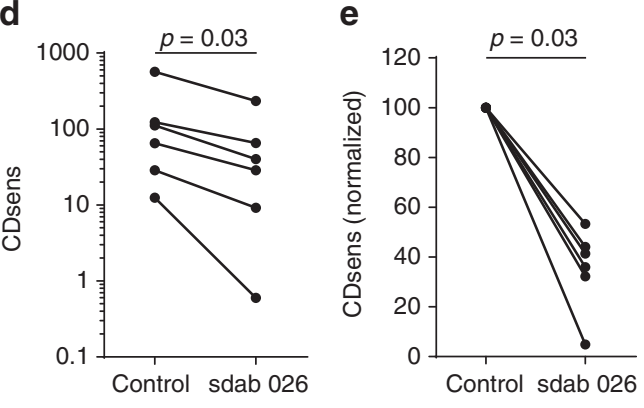

f

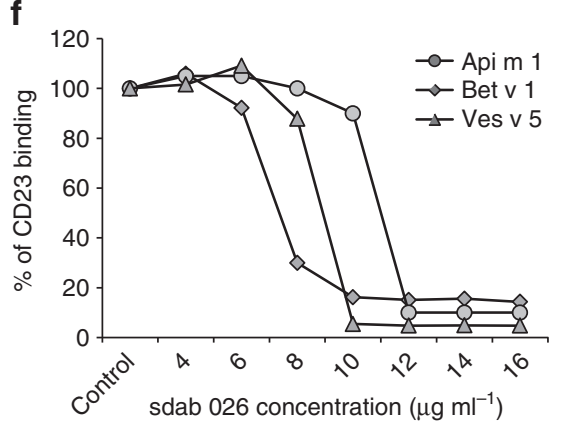

g

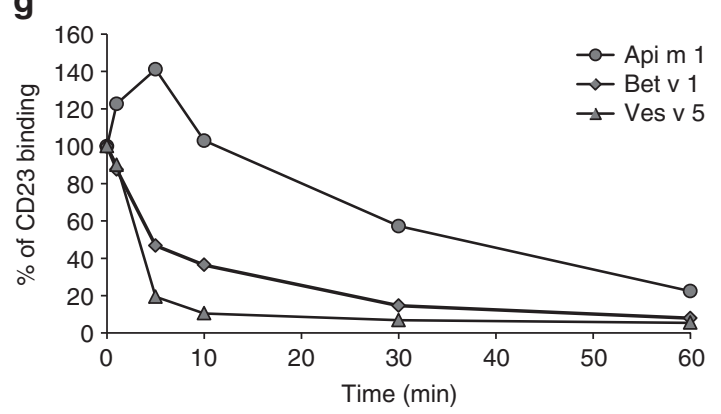

Fig. 4 Functional activity of the sdab. a Inhibition of mediator release from effector cells by 026 sdab was assessed in RBL-SX38 cells providing the human FceRI. Cells were sensitized with IgE Fc in presence of decreasing amounts of $026 \mathrm{sdab}$. Degranulation was induced by anti-lgE antibody and monitored by released $\beta$-hexosaminidase activity. Data are the mean \pm standard deviation of triplicate measurements. $\mathbf{b}$ Removal of IgE from the surface of basophils by the $026 \mathrm{sdab}$, the inactive sdab mutant 112 and omalizumab was analyzed by surface staining of IgE using flow cytometry. Basophils of three patients with allergy to different major allergens were used. Concentration of omalizumab was identical to sdab concentrations, but doubled to $90 \mu$ mol $\mathrm{I}^{-1}$ in the prolonged periods. c The capability to reduce basophil sensitivity by displacement of IgE from FceRI was assessed by basophil activation test (BAT). Basophils were incubated with 026 sdab followed by incubation with Bet $v 1$. Activation of basophils was then assessed by detecting $C D 63^{+}$basophils in flow cytometry (exemplary BAT of one donor included in panel d). Data are mean \pm s.d. d The effect of the 026 sdab on basophil activation was analyzed for six birch pollen-sensitized patients. Reduction of effector cell sensitivity was evaluated by CDsens analysis. Comparison of paired samples \pm sdab 026 treatment was done by using the nonparametric Wilcoxon signed-rank test. Differences were considered statistically significant at $p$ values $<0.05$.

e Inhibition of IgE binding to CD23 by 026 sdab analyzed by ELIFAB. The binding of allergen:IgE complexes to surface bound CD23 was detected by anti-IgE antibodies. Complexes were formed using sera of allergic patients having highly elevated specific IgE to Api $\mathrm{m} \mathrm{1}$, Bet $\vee 1$ and $\mathrm{Ves} \vee 5$ ( $>100 \mathrm{kUA} \mathrm{I}^{-1}$ ), respectively. $\mathbf{f}$ The displacement of preformed IgE:allergen complexes from CD23 using sera as in panel e by the sdab was analyzed by detecting remaining binding of allergen:lgE complexes after incubation with $026 \mathrm{sdab}$

$\left(>100 \mathrm{kUA}^{-1}\right)$ against the honeybee venom allergen Api $\mathrm{m} \mathrm{1}$, the birch pollen allergen Bet $\mathrm{v} 1$ and the major yellow jacket venom allergen Ves v 5 were incubated with the respective allergen and the IgE:allergen complexes applied to CD23. The sdab efficiently prevented binding of the IgE:allergen complexes to CD23 (Fig. 4f). Moreover, even when applied after binding of the complexes to CD23, the sdab displaced IgE efficiently within minutes (Fig. 4g).
SAXS analysis of single-domain antibody binding to IgE Fc. We next asked how sdab binding influences the conformation of an $\operatorname{IgE} \mathrm{Fc}$ including the $\mathrm{C} \varepsilon 2$ domain. Contaminating $\operatorname{IgE} \mathrm{Fc}$ Ce3-4 was first removed by hydrophobic interaction chromatography after which the IgE Fc:026 sdab complex was isolated by size-exclusion chromatography (Supplementary Figure 9). Synchrotron small-angle X-ray scattering (SAXS) data were then collected for both unbound IgE Fc and in complex with 026 sdab 
a

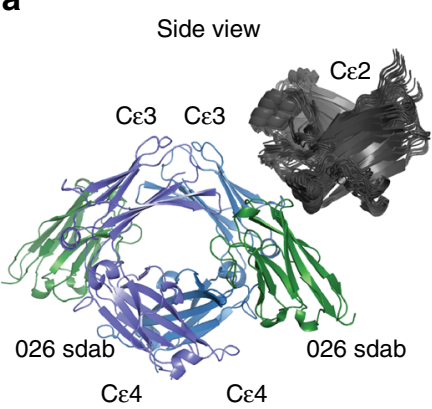

C

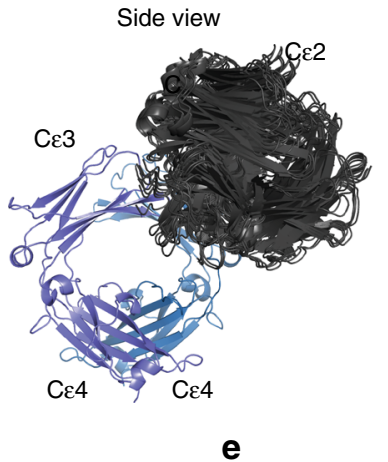

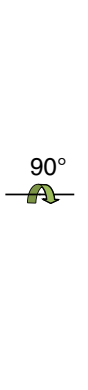

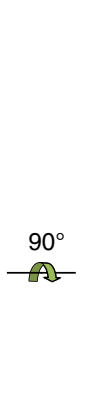

$90^{\circ}$

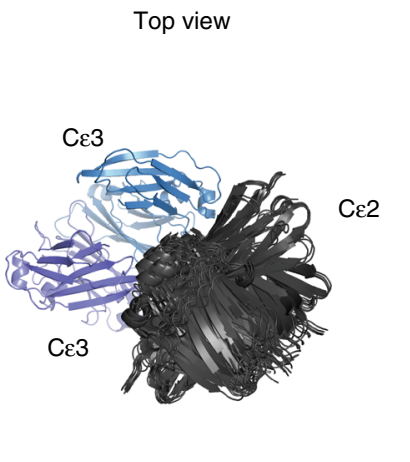

b
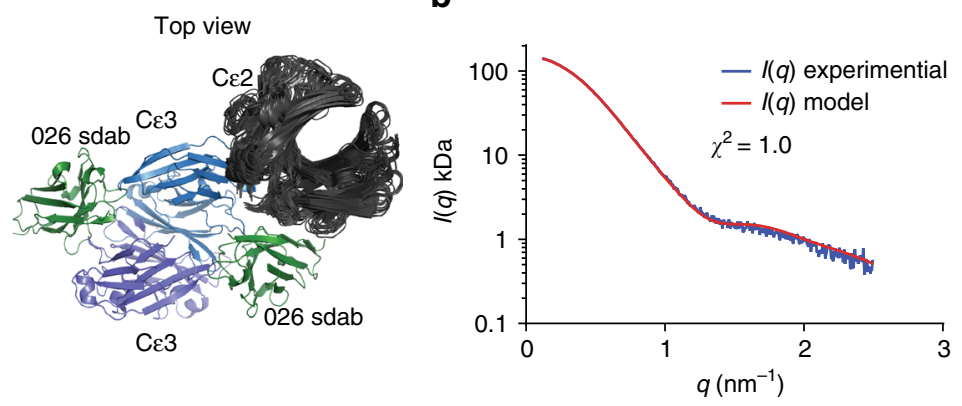

d

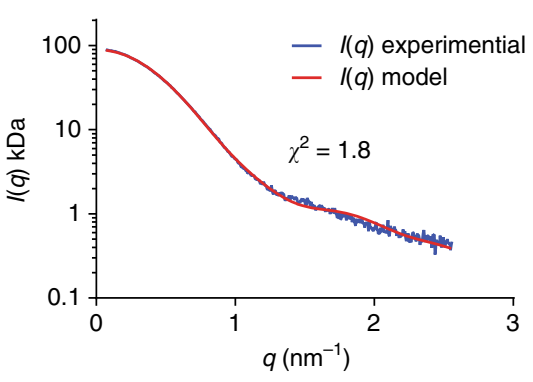

$\mathbf{f}$

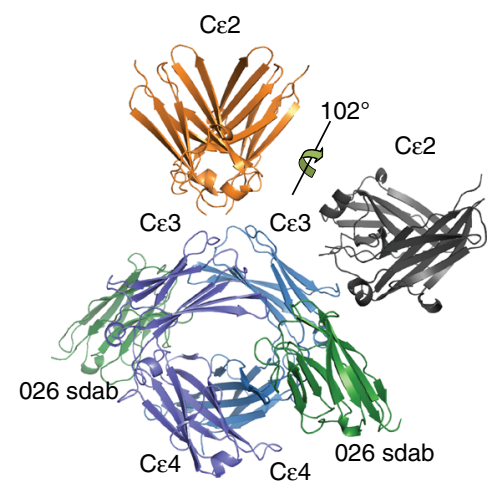

Fig. 5 SAXS analysis of the IgE Fc:026 sdab complex and unbound IgE Fc. a Side and top views of 20 superimposed rigid body models obtained with 026 in green, the $C_{\varepsilon} 3$ and $C_{\varepsilon} 4$ domains in blue, and the $C_{\varepsilon 2}$ domains in gray. $\mathbf{b}$ The fit of the scattering curve calculated from a representative rigid body model to the experimental curve as output from CORAL. c, $\mathbf{d}$ Same as panels $\mathbf{a}, \mathbf{b}$, but for the unbound Fc with the C 33 domains in the closed conformation. $\mathbf{e}$ In the fully bent conformation of free IgE Fc (PDB ID: 2WQR) the C 2 domains (orange) would substantially overlap with the location of sdab 026. $\mathbf{f}$ Comparison of the extended IgE Fc conformation ( $\mathrm{C} \varepsilon 2$ in orange, PDB ID: 4J4P) and the half-bent conformation of the Fc in complex with sdab 026 observed by SAXS (Ce2 in gray)

(Supplementary Figure 10a-d, Supplementary Table 2). The pair-distance distribution plots showed very similar mass distributions in the two cases but with the IgE Fc:026 sdab complex being slightly larger (Supplementary Figure 10e). Rigid body modeling of the IgE Fc:026 sdab complex with the C 83 domains fixed in the closed conformation as in our crystal structure, resulted in a tightly clustered ensemble of solutions fitting the SAXS curve well with an average $\chi^{2}=1.06 \pm 0.04$ according to CORAL (Fig. 5a, b). Likewise, we conducted rigid body refinement against the SAXS data collected from unbound IgE Fc with the C\&3 domains fixed in either the closed or the open conformation during refinement. Both scenarios resulted in solutions fitting the data with $\chi^{2}$ values in the range $1.8-2.3$ according to CORAL. Qualitatively output models from both scenarios were in the bent conformation with the C $\varepsilon 2$ domains located toward the C 83 domains (Fig. 5c, d). In roughly 95\% of the output models with the Ce3 domains fixed in the closed conformation, a substantial overlap between the Ce2 domains and a bound sdab 026 would occur. In agreement with this, a comparison of our crystal structure of the IgE Fc:026 sdab complex not containing the Ce2 domains with the crystal structure of unbound IgE Fc (pdb ID: $2 \mathrm{WQR})^{21}$ reveals that in the sdab complex, IgE Fc must adopt a significantly less bent conformation than that observed in the crystal structure of unbound IgE Fc to avoid overlap between the $\mathrm{C} \varepsilon 2$ domains and bound sdab (Fig. 5e). Hence, the rigid body modeling provided evidence for a sdab-mediated displacement of the $\mathrm{C} \varepsilon 2$ and unbending of the $\operatorname{IgE} \mathrm{Fc}$ resulting in a so far undescribed conformation of the Fc Ce2-4 domains. But an $\sim 100^{\circ}$ rotation would still be required to reach the fully extended conformation of IgE Fc (Fig. 5f).

To investigate whether the SAXS data for $\operatorname{IgE} \mathrm{Fc}$ and $\operatorname{IgE}$ Fc:026 sdab complex could be better explained by a mixture of conformations, we also employed the ensemble optimization method $(\mathrm{EOM})^{33}$. For the IgE Fc:026 sdab complex the selected structures clustered around a higher $D_{\max }$ than the average generated structure (Supplementary Figure 11a). The two representative structures adopted different bent conformations. The conformation representing $63 \%$ of the scattering was almost 
identical to the rigid body structures output from CORAL, whereas the second conformation was slightly more extended. The ensemble fitted the experimental data with $\chi^{2}=1.27$ as calculated by GAJOE (Supplementary Figure 11b). The corresponding EOM analysis of the unbound IgE Fc SAXS data resulted in ensemble optimized structures falling in two separate clusters with either a significantly smaller $D_{\max }$ or a significantly larger $D_{\max }$ than the average generated structure (Supplementary Figure 11c). The ensemble fitted the experimental data with $\chi^{2}=2.43$ (Supplementary Figure 11d). The low $D_{\max }$ cluster accounting for $\sim 70 \%$ of the scattering corresponded to a bent conformation similar to the CORAL rigid body solutions, whereas the high $D_{\max }$ cluster corresponded to a conformation falling in between the fully extended and bent and accounted for $\sim 30 \%$ of the total scattering. Overall, the EOM analysis of the solution scattering data confirmed the models obtained by rigid body refinement but in addition provided evidence of a second conformation in both cases. Altogether our SAXS analysis suggested that $\operatorname{IgE} \mathrm{Fc}$ in solution mainly adopts the bent conformation as anticipated and that binding of $026 \mathrm{sdab}$ induces IgE Fc unbending to a conformation that is in between the classical bent and the fully extended two-fold symmetric conformation.

\section{Discussion}

Key to intervention in allergic diseases is the reduction of $\operatorname{IgE}$ in the serum and eventually on effector cells. In this study, we show that the anti-IgE sdab 026 targets $\operatorname{IgE}$ by binding to an epitope within the Fc domains that does not significantly overlap with the FceRI-binding site. Notably the epitope is a conformational epitope involving two domains and a buried surface of $\sim 800 \AA^{2}$ that is larger than the omalizumab epitope ${ }^{23}$. Both epitope and inhibition mode of sdab 026 are different from those previously observed for anti-IgE molecules. The sdab blocks interaction with FceRI by trapping IgE Fc in a closed conformation of the Ce3 domains incompatible with FceRI binding and a slightly more extended conformation of the Ce2 domains.

The sdab epitope shows significant overlap with the CD23binding site. Although additional importance of the stalk region of CD23 in binding to IgE has been postulated ${ }^{34}$ the shared motif of the sdab and CD23 epitopes and the high affinity of the $026 \mathrm{sdab}$ render an effect of the stalk on the inhibitory activity of the sdab unlikely. As observed in the ELIFAB analyses the inhibition of IgE binding to CD23 and displacement of IgE from CD23 strongly supports the epitope inferred from the structure and the mode of action of the anti-IgE sdab. Recently it has been verified that omalizumab also blocks the interaction with $\mathrm{CD} 23^{34}$, most likely due to minor overlap of the epitope with the binding site ${ }^{23}$.

Binding of allergen:IgE complexes to CD23, a C-type lectin and low affinity IgE receptor, is crucial for facilitated antigen presentation and transport across the epithelium, and amplifies the generation and epitope spread of specific $\operatorname{IgE}^{2,35}$. The inhibition of CD23 binding by patients' serum IgG correlates with the activity of blocking IgG induced by successful allergen immunotherapy ${ }^{36}$ and leads to reduced T-cell activation ${ }^{37}$. Combining immuno-therapy with omalizumab results in clear benefits for treatment efficacy ${ }^{38}$. The main target of anti-IgE approaches however remains interference with FceRI-mediated immediate effects.

IgE Fc adopts conformational states within the Ce3-Ce4 domain pairs ranging from an open conformation when in complex with FceRI to the closed conformation when bound to $\mathrm{CD} 23$. Binding of FceRI and CD23 to IgE is mutually exclusive as each is dependent on the conformation of Fc. FceRI binding to free IgE moves the two C 83 domains away from each other, decreases the angle between $\mathrm{C} \varepsilon 3$ and $\mathrm{C} \varepsilon 4$ and makes the binding site for CD23 inaccessible. Conversely, binding of CD23 to free IgE increases the angle between $\mathrm{C} \varepsilon 3$ and $\mathrm{C} \varepsilon 4$, moves the $\mathrm{C} \varepsilon 3$ domains to a more closed conformation and prevents IgE Fc interactions with FceRI. In complex with the sdab, the $\mathrm{C} \varepsilon 3$ and C\&4 domains adopt the closed conformation, which is incompatible with FceRI binding. CD23 is contacting residues in Ce3 and $\mathrm{C} \varepsilon 4$ within the same chain and enforces the closed conformation by pushing the C 83 domains closer together. With a similar result, the sdab binds $\mathrm{C} \varepsilon 3$ and $\mathrm{C} \varepsilon 4$ from different $\varepsilon$ chains forcing a closed conformation by pulling the $\mathrm{C} \varepsilon 3$ from one chain toward the other.

Most of the structures available for IgE Fc contain only the CE3-4 domains, and likewise we did not obtain crystals with the entire IgE Fc probably since the flexibility of the Ce2 domains in the IgE Fc hampers crystallization. The C $83-4$ fragment is often used in a stabilized form having artificial disulfide bridges or reduced glycosylation as a result of mutagenesis or choice of the expression host. Here we use the authentic IgE Fc and thereby minimize the risk of trapping the molecules in a rare or artificial conformation.

Being unable to crystallize sdab in complex with the full IgE Fc we used SAXS to analyze the complex and free IgE Fc in solution. This allowed insights into the positioning of the CE2 domain in solution for both the free Fc as well as in the complex with the sdab. Two different approaches to SAXS based modeling suggested that with respect to the sdab complex, the IgE Fc Ce2 domains appear to adopt a primary conformation in between the two well-established bent and extended IgE Fc conformations. In unbound $\operatorname{IgE} \mathrm{Fc}$, the dominating conformation appears to be the bent conformation. In addition, EOM analysis suggested that one minor conformation somewhat different from the major conformation may be present in both cases.

Recently, the displacement of IgE from FceRI by a DARPin IgE antagonist was described ${ }^{23,31}$, and a similar, but lower activity has been reported for omalizumab. Our data suggest that the $026 \mathrm{sdab}$ displaces IgE from FceRI more efficiently than omalizumab. An even four-fold molar excess of omalizumab Fab fragment over the sdab did not result in significant IgE displacement. Apparently, the sdab removes IgE from the surface of effector cells as shown for basophils obtained from patients with different types of allergies. The reduction of IgE correlates with a decreased sensitivity of the effector cells to allergen-dependent activation as shown in a cohort of birch pollen-sensitized patients. It is imperative to consider that sensitivity of effector cells is shaped by the characteristics of surface $\operatorname{IgE}$, e.g., ratio of specific and total IgE, affinity and repertoire complexity $^{39}$. Although we used the dominant birch pollen allergen, Bet $\mathrm{v} 1$, patient-specific sensitization profiles influence the reduction of cellular sensitivity, an effect likely to be even more pronounced in multisensitized individuals.

With respect to the accessibility of one sdab-binding site on the IgE even when bound to the FceRI, the disruptive activity of the sdab is most likely driven by the conformational rearrangement upon sdab binding to the FceRI bound IgE. With the capability of the E2_79 DARPin, omalizumab and the 026 sdab for displacing $\operatorname{IgE}$ it appears reasonable that the majority of anti-IgE molecules might exhibit similar activity, at least to a certain extent. Increasing this activity could become an important strategy for improving efficacy of anti-IgE therapeutics ${ }^{40}$. Although the removal of IgE from both circulation and effector cells could represent a benefit, the in vivo effect has not been fully proven yet. It has been shown that reduction of IgE on effector cells is counterbalanced by increased sensitivity ${ }^{41}$. Additional studies are therefore needed to address the displacement of $\operatorname{IgE}$ and its consequences and implications in detail. 
Single-domain antibodies exhibit high production yield in simple expression systems and extraordinary stability, properties that render them interesting for numerous biotechnological and biomedical applications ${ }^{42}$. Targeting IgE with sdabs could offer a variety of benefits. Their 10 -fold smaller size as compared to IgG allows efficient targeting of less accessible sites and the monovalent format prevents formation of larger complexes. State-ofthe-art strategies for half-life extension and multiple targeting can easily be applied to sdabs. Delivery in functional form via mucosal and airway tissues is possible using sdabs ${ }^{43}$ and could improve the use of difficult routes for anti-IgE application ${ }^{44}$.

The sdab's mode of action identified in this study could also open up for the development of novel anti-IgE molecules of even lower molecular mass. It seems reasonable that high affinity targeting of an epitope similar to the sdab epitope by smaller molecules might drive similar conformational rearrangements. Thus, our description of the 026 sdab mode of action is likely to accelerate the development of anti-allergy and asthma drugs in the future.

\section{Methods}

Crystallization. The 026 sdab:IgE Fc complex was concentrated to $5 \mathrm{mg} \mathrm{ml}^{-1}$ in $20 \mathrm{mM}$ HEPES, $50 \mathrm{mM} \mathrm{NaCl}, \mathrm{pH} 7.2$, and crystallized by vapor diffusion in sitting drops formed by mixing protein and reservoir solution containing $0.1 \mathrm{M}$ imidazole $\mathrm{pH} 7.0,11-12 \%$ polyethylene glycol (PEG) 20,000 in the ratio 1:1. Crystals were cryocooled in liquid nitrogen after transfer to a cryo-protectant composed of $0.1 \mathrm{M}$ imidazole $\mathrm{pH}$ 7.0, 12\% w/v PEG 20,000 and 30\% glycerol.

Data collection and structure determination and refinement. Data were collected at Diamond $124^{45}$ and processed with $\mathrm{XDS}^{46}$. The structure was determined by molecular replacement with PHASER ${ }^{47}$ using the pdb ID: $2 \mathrm{WQR}$ as search model $^{21}$. The model was rebuild in $\mathrm{Coot}^{48}$ and refined with Phenix.refine and $\mathrm{iMDFF}^{49,50}$. Figures were prepared with the PyMOL Molecular Graphics System (Schrödinger LLC).

Protein expression and purification. The IgE $F_{c}$ constant region cDNA was obtained from an IgE expression vector initially constructed from a human cDNA library. The cDNA was introduced into an expression vector providing a human immunoglobulin signal sequence via SmiI and MssI restriction enzymes ${ }^{51}$. Point mutations were introduced by PCR Human embryonic kidney cells (HEK-293, ATCC) were cultivated in Dulbecco's modified Eagle medium (DMEM) supplemented with $10 \%(\mathrm{v} / \mathrm{v})$ heat-inactivated fetal calf serum (FCS), $100 \mathrm{IU} \mathrm{ml}^{-1}$ penicillin and $100 \mu \mathrm{g} \mathrm{ml}^{-1}$ streptomycin. HEK-293 cells were transfected with the expression vector using Nanofectin (GE Healthcare) according to the recommendations of the manufacturer. After selection by addition of zeocin stably transfected cells showed an expression yield of 10-15 $\mathrm{mgl}^{-1}$. Cell culture supernatant obtained in roller flasks (Greiner) was collected and subsequently subjected to purification of the $\operatorname{IgE} \mathrm{Fc}$ via nickel based affinity chromatography. The supernatant was loaded on a $1 \mathrm{ml}$ HisTrap excel column (GE Healthcare) equilibrated with PBS (500 $\mathrm{mM}$ $\mathrm{NaCl}, 40 \mathrm{mM} \mathrm{Na}_{2} \mathrm{HPO}_{4}, 10 \mathrm{mM} \mathrm{NaH} \mathrm{PO}_{4}, \mathrm{pH}$ 7.4). After washing with 10 column volumes (CV) of PBS and $20 \mathrm{CV}$ of $5 \% \mathrm{PBS} /$ imidazole $(100 \mathrm{mM} \mathrm{NaCl}, 40 \mathrm{mM}$ $\mathrm{Na}_{2} \mathrm{HPO}_{4}, 10 \mathrm{mM} \mathrm{NaH}_{2} \mathrm{PO} 4,300 \mathrm{mM}$ imidazole, $\mathrm{pH}$ 7.4) the IgE Fc was eluted in a $5-100 \%$ gradient of PBS/imidazole. Pooled fractions were dialyzed against Buffer A (20 mM NaOAc, $50 \mathrm{mM} \mathrm{NaCl}, \mathrm{pH} 6.5)$ and subsequently applied to a $1 \mathrm{ml}$ Mono S 5/50 column (GE Healthcare) equilibrated with Buffer A. After washing with Buffer A, the protein was eluted with $20 \mathrm{CV}$ of Buffer B $(20 \mathrm{mM} \mathrm{NaOAc}, 1 \mathrm{M} \mathrm{NaCl}$, $\mathrm{pH} 6.5$ ) with a $0-50 \%$ gradient. The IgE $\mathrm{Fc}$ was further purified by size exclusion chromatography using a $24 \mathrm{ml}$ Superdex $20020 / 300 \mathrm{GL}$ column (GE Healthcare) equilibrated in $20 \mathrm{mM}$ HEPES, $50 \mathrm{mM} \mathrm{NaCl}, \mathrm{pH} 7.2$.

The DNA of the sdab 026 (patent WO 2012'/175740 A1) was obtained as a synthetic gene and cloned into the bacterial expression vector pET22+ providing a pelB signal sequence and a C-terminal histidine tag (Supplementary Table 3). Expression of the sdab 026 and its variants was performed for $3 \mathrm{~h}$ at $30^{\circ} \mathrm{C}$ after IPTG induction. The supernatant was subjected to nickel-based affinity chromatography on a $1 \mathrm{ml}$ HisTrap excel column (GE Healthcare) equilibrated with PBS. After washing with $10 \mathrm{CV}$ of PBS and $20 \mathrm{CV}$ of $5 \% \mathrm{PBS} / 300 \mathrm{mM}$ imidazole the protein was eluted with a $5 \mathrm{CV}$ gradient from $5-100 \%$ of PBS/ imidazole. The sdab mutants were purified accordingly. After dialysis against Buffer A (20 mM Tris, $50 \mathrm{mM} \mathrm{NaCl}$, pH 8.5) the sdab was further purified using ion exchange chromatography using a $6 \mathrm{ml}$ Resource Q column (GE Healthcare) equilibrated in Buffer A. After washing with $5 \mathrm{CV}$ of Buffer A, the protein was eluted with a 5 CV gradient from $0-50 \%$ Buffer B $(20 \mathrm{mM}$ Tris, $1 \mathrm{M} \mathrm{NaCl}, \mathrm{pH} 8.5)$.

Prior to SAXS data collection IgE Fc was further purified to remove the CE3-4 fragment by hydrophobic interaction chromatography using a $1 \mathrm{ml}$ Source 15PHE column (GE Healthcare) equilibrated in Buffer A (1.6 $\mathrm{M} \mathrm{NH}_{3} \mathrm{SO}_{4}, 20 \mathrm{mM}$ HEPES,
$\mathrm{pH}$ 7.2). The sample obtained from size exclusion chromatography was dialyzed against Buffer A and applied to the column. After washing with $5 \mathrm{CV}$ of Buffer A, the protein was eluted with a $15 \mathrm{CV}$ gradient from $0-100 \%$ Buffer B $(50 \mathrm{mM} \mathrm{NaCl}$, $20 \mathrm{mM}$ HEPES, pH 7.2). IgE Fc Ce2-4 from the Source 15Phe purification was mixed with purified $026 \mathrm{sdab}$ and the complex was purified by size-exclusion chromatography as described above.

Characterization of IgE Fc and 026. The binding of $026 \mathrm{sdab}$ to IgE Fc was assessed in ELISA. Purified IgE Fc $\left(50 \mu \mathrm{g} \mathrm{ml}^{-1}\right)$ was coated on microtiter plates (Greiner) at $4^{\circ} \mathrm{C}$ and blocked with $40 \mathrm{mg} \mathrm{ml}^{-1}$ milk powder in PBS. Thereafter, $026 \mathrm{sdab}$ at a concentration of $10 \mu \mathrm{g} \mathrm{ml}^{-1}$ was incubated for $2 \mathrm{~h}$ at RT. After washing, binding to $\mathrm{IgE} \mathrm{Fc}$ was detected using alkaline phosphatase-conjugated anti-human kappa antibody and anti-human IgG antibody diluted 1:30,000 and 30 $\mu \mathrm{l}$ of substrate solution.

For comparative assessment of anti-IgE antibodies, ELISA microtiter plates were coated with $026 \mathrm{sdab}\left(25 \mathrm{\mu g} \mathrm{ml}^{-1}\right)$ at $4{ }^{\circ} \mathrm{C}$ and blocked with $40 \mathrm{mg} \mathrm{ml}^{-1}$ milk powder in PBS. After incubation with $\operatorname{IgE} \mathrm{Fc}$, the anti-IgE antibodies were incubated in a final volume of $20 \mu \mathrm{l}$ for $4 \mathrm{~h}$ at room temperature. After washing bound antibodies were detected using an alkaline phosphatase-conjugated antihuman IgG antibody diluted 1:20,000, and $30 \mu \mathrm{l}$ of substrate solution $\left(5 \mathrm{mg} \mathrm{ml}^{-1} 4\right.$ nitro-phenylphosphate, AppliChem).

Affinity measurements. The affinity of the sdab was determined by using a T200 Biacore system. IgE Fc was immobilized to a total of 110 resonance units (RU) onto a CM5 sensor chip (GE Healthcare) using NHS/EDC coupling procedures. Analyses were performed at $25^{\circ} \mathrm{C}$ in a buffer containing $10 \mathrm{mM}$ monosodium phosphate, $40 \mathrm{mM}$ disodium phosphate and $100 \mathrm{mM} \mathrm{NaCl}, \mathrm{pH} 7.4$ supplemented with $0.01 \%$ Tween-20. For kinetic analyses the 026 sdab was injected at concentrations from 0.003 to $50 \mu \mathrm{g} \mathrm{ml}^{-1}$ at a flow rate of $25 \mu \mathrm{min}^{-1}$. Association and dissociation were assessed for $600 \mathrm{~s}$. Regeneration of sensor surfaces was performed by subsequent injection of $1 \mathrm{M}$ Tris buffer, $\mathrm{pH} 10$. The dissociation constant at equilibrium $K_{\mathrm{D}}$ was calculated using a 1:1 binding model and the Biacore T200 evaluation software.

Basophil activation tests and mediator release assays. Peripheral EDTA blood from allergic donors was preincubated with 026 sdab, mul12 or omalizumab (Novartis) at concentrations from 0 to a maximum of $45 \mu \mathrm{mol} \mathrm{l}^{-1}$ (sdab 026 and mul12) and from 0 to $90 \mu \mathrm{moll}^{-1}$ (omalizumab), respectively, in IL-3-containing stimulation buffer (Bühlmann) for up to $60 \mathrm{~min}$ on a shaker. Samples were stained with anti-CCR3-PE in a $37^{\circ} \mathrm{C}$ water bath for $15 \mathrm{~min}$ and anti-IgE-FITC (both BioLegend) for $20 \mathrm{~min}$ at $4{ }^{\circ} \mathrm{C}$. Erythrocytes were lysed for $7 \mathrm{~min}$ followed by centrifugation at $500 \times g$ for $5 \mathrm{~min}$. Cell pellets were resuspended in $100 \mu \mathrm{l}$ washing buffer and the mean fluorescence intensity (MFI) of IgE on $\mathrm{CCR}^{+}{ }^{+} \mathrm{SSC}^{\text {low }}$ basophils was measured by flow cytometry (FACS Calibur, BD Biosciences).

To analyze the impact of IgE026 on basophil activation, peripheral EDTA blood from six patients with birch pollen allergy was incubated $\pm 45 \mu \mathrm{moll}^{-1}$ sdab 026 for $60 \mathrm{~min}$ on a shaker. Afterwards, Bet v 1 (Biomay) diluted at different concentrations $\left(0.01-1000 \mathrm{ng} \mathrm{ml}^{-1}\right)$ in stimulation buffer was added. In each case one sample was left unstimulated to exclude background activation. Next, all samples were incubated with $15 \mu \mathrm{l}$ of staining reagent (Bühlmann) consisting of anti-CCR3-PE and anti-CD63-FITC in a $37^{\circ} \mathrm{C}$ water bath for $15 \mathrm{~min}$. As above, erythrocytes were lysed for $7 \mathrm{~min}$ followed by centrifugation at $500 \times g$ for $5 \mathrm{~min}$. Cell pellets were resuspended in $100 \mu \mathrm{l}$ washing buffer and basophil activation was measured by flow cytometry (FACS Calibur, BD Biosciences). Basophils were identified as $\mathrm{CCR} 3^{+} \mathrm{SSC}^{\text {low }}$ cells and $\mathrm{CD}^{+} 3^{+}$basophils were considered as activated. All results were analyzed using BD FACSDiva software (BD Biosciences).

CDsens analyses were performed as basophil allergen threshold stimulation. Basophil allergen sensitivity was measured as the allergen concentration eliciting $50 \%$ (EC50) of maximum CD63 upregulation. The CDsens is defined as inverted value for EC50 multiplied by 100, and was calculated by the following formula: CDsens $=1 / \mathrm{EC} 50 \times 100^{52}$

In order to analyze in vitro degranulation RBL-SX38 cells (kindly provided by J.P. Kinet) were sensitized with $\mathrm{rIgE} \mathrm{Fc}^{53}$. After washing with incomplete Tyrode's buffer, receptor cross-linking was performed by incubation with goat anti-human $\mathrm{IgE}$ for $60 \mathrm{~min}$ at $37^{\circ} \mathrm{C}$. Release of $\beta$-hexosaminidase from viable vs. lysed cells was assessed for $60 \mathrm{~min}$ at $37^{\circ} \mathrm{C}$ using p-nitrophenyl $\mathrm{N}$-acetyl-glucosaminide (SigmaAldrich) as a substrate. After stopping the reaction by addition of carbonate buffer $(0.1 \mathrm{M}, \mathrm{pH} 10)$ the absorbance was recorded at $405 \mathrm{~nm}$.

Analyses of CD23 binding using the ELIFAB assay. In order to evaluate the capability of 026 sdab to inhibit the binding of IgE:allergen complexes to CD23 the ELISA-based ELIFAB assay, a cell-free variant of the FAB assay, was performed $^{32,54}$. To allow for formation of IgE:allergen complexes $20 \mu \mathrm{l}$ of indicator serum containing either high Api m 1-, Bet v 1- or Ves v 5-specific IgE concentrations (all $>100 \mathrm{kUA} \mathrm{l}^{-1}$ ) was preincubated with the respective allergen at $37^{\circ}$ $\mathrm{C}$ for $1 \mathrm{~h}$ in the presence of different 026 sdab concentrations. Following preincubation, IgE:allergen complexes were transferred to plates coated with CD23 (R\&D Systems, Bio-Techne) and incubated for $1 \mathrm{~h}$ at RT. IgE:allergen complexes bound to immobilized CD23 were detected by adding biotin-conjugated anti- 
human IgE antibody (BD Biosciences), streptavidin-peroxidase (Sigma-Aldrich), and 3,3',5,5'-tetramethylbenzidine (TMB) substrate (Calbiochem, Merck Millipore). All samples were analyzed in duplicates.

In a second set of ELIFAB experiments, preformed IgE:allergen complexes bound to $\mathrm{CD} 23$ were incubated with $5 \mu \mathrm{l} 026$ sdab up to 60 min evaluating the ability of $026 \mathrm{sdab}$ to displace IgE:allergen complexes from CD23. Displacing IgE: allergen complexes from immobilized CD23 was determined at a 026 sdab concentration of $12 \mu \mathrm{g} \mathrm{ml}^{-1}$ for Api m 1 (Latoxan), $8 \mu \mathrm{g} \mathrm{ml}^{-1}$ for Bet $\mathrm{v} 1$ (Biomay) and $11 \mu \mathrm{g} \mathrm{ml}^{-1}$ for Ves $\mathrm{v}$, respectively.

Small-angle X-ray scattering data collection and modeling. The SAXS measurements of IgE Fc and IgE Fc in complex with sdab 026 were performed in batch mode at the BM29 beamline at the European Synchrotron Radiation Facility (ESRF), Grenoble, France ${ }^{55}$. The data were collected using a PILATUS $1 \mathrm{M}$ pixel detector and $\lambda=0.992 \AA$ in a temperature controlled capillary at $4{ }^{\circ} \mathrm{C}$. The sampleto-detector distance was $2.872 \mathrm{~m}$, covering a range of momentum transfer $0.04<q$ $<5 \mathrm{~nm}^{-1}(q=(4 \times \pi \times \sin \theta) \div \lambda$, where $2 \theta$ is the scattering angle). Samples in $20 \mathrm{mM}$ HEPES, $50 \mathrm{mM} \mathrm{NaCl}, \mathrm{pH} 7.2$ were investigated in the concentration ranges 0.5-1.7 and 2.2-9.0 $\mathrm{mg} \mathrm{ml}^{-1}$ for the IgE Fc:026 sdab complex and IgE Fc, respectively. Data were collected with 10 exposures of $2 \mathrm{~s}$. Radial averaging, buffer subtraction and concentration scaling were performed using the beamline pipeline ${ }^{56}$. Bovine serum albumin (Sigma, A7638-5GP) used for calculation of the molecular weight (Supplementary Table 1) was solubilized in a buffer containing $50 \mathrm{mM}$ Hepes pH 7.5 at concentration of $5 \mathrm{mg} \mathrm{ml}^{-1}$. For the IgE Fc:026 sdab complex a concentration of $1.7 \mathrm{mg} \mathrm{ml}^{-1}$ was used for further modeling. For $\mathrm{IgE} \mathrm{Fc}$ the measurements at 2.2 and $9.0 \mathrm{mg} \mathrm{m}^{-1}$ were merged using ALMERGE ${ }^{57}$. The pair distribution function was calculated by indirect Fourier Transform using $\mathrm{GNOM}^{58}$. Rigid body refinements were performed using a momentum transfer range of $q<3.0 \mathrm{~nm}^{-1}$ with CORAL ${ }^{59}$. The $026 \mathrm{sdab}$ and the $\mathrm{Fc}_{\mathrm{c}} \mathrm{C} \varepsilon 3$ and $\mathrm{C} \varepsilon 4$ domains were used as a single rigid body taken directly from our crystal structure. The two C 22 domains from the pdb entry $2 \mathrm{WQR}$ were grouped into a single rigid body, and the C-terminal of both of the Ce2 domains were linked to the $\mathrm{N}$ terminal residue of their corresponding $\mathrm{C} \varepsilon 3$ domain with a distance restraint of 25 A. Calculation of the angle of rotation between the SAXS models and the extended (RCSB: 4J4P) and bent (RCSB: $2 \mathrm{WQR}$ ) conformation of IgE Fc was performed using DynDom ${ }^{60}$. Ensemble optimization of both unbound IgE Fc and the IgE Fc:026 sdab complex was performed using the EOM 2.0 programs RANCH and GAJOE $^{33}$. A total of 5000 models were generated utilizing the same rigid bodies as for CORAL rigid body modeling, but with a randomly generated alpha carbon trace connecting the two rigid bodies, instead of a distance restrain. GAJOE was then used to select the ensemble of models best representing the scattering curve.

Patient material. The study was approved by the Ethics Committee of the Medical Faculty of Philipps University, Marburg, Germany; all patients provided written informed consent to participate in the trial.

Data availability. Coordinates and structure factors have been deposited in the Protein Data Bank under accession code 5NQW. Other data are available from the corresponding authors upon reasonable request.

Received: 2 June 2017 Accepted: 20 November 2017

Published online: 02 January 2018

\section{References}

1. Finkelman, F. D., Boyce, J. A., Vercelli, D. \& Rothenberg, M. E. Key advances in mechanisms of asthma, allergy, and immunology in 2009. J. Allergy Clin. Immunol. 125, 312-318 (2010).

2. Gould, H. J. \& Sutton, B. J. IgE in allergy and asthma today. Nat. Rev. Immunol. 8, 205-217 (2008).

3. Iikura, M. et al. Regulation of surface FcepsilonRI expression on human eosinophils by IL-4 and IgE. Int. Arch. Allergy Immunol. 124, 470-477 (2001).

4. Kinet, J. P. The high-affinity IgE receptor (Fc epsilon RI): from physiology to pathology. Annu. Rev. Immunol. 17, 931-972 (1999).

5. Kraft, S. \& Kinet, J. P. New developments in FcepsilonRI regulation, function and inhibition. Nat. Rev. Immunol. 7, 365-378 (2007).

6. Chang, T. W. The pharmacological basis of anti-IgE therapy. Nat. Biotechnol. 18, 157-162 (2000).

7. Peng, Z. Vaccines targeting IgE in the treatment of asthma and allergy. Hum. Vaccin. 5, 302-309 (2009)

8. Presta, L. et al. The binding site on human immunoglobulin $\mathrm{E}$ for its high affinity receptor. J. Biol. Chem. 269, 26368-26373 (1994).

9. Presta, L. G. et al. Humanization of an antibody directed against IgE. J. Immunol. 151, 2623-2632 (1993).

10. Shields, R. L. et al. Inhibition of allergic reactions with antibodies to IgE. Int. Arch. Allergy Immunol. 107, 308-312 (1995).
11. MacGlashan, D. W. Jr et al. Down-regulation of Fc(epsilon)RI expression on human basophils during in vivo treatment of atopic patients with anti-IgE antibody. J. Immunol. 158, 1438-1445 (1997).

12. Gomez, G., Jogie-Brahim, S., Shima, M. \& Schwartz, L. B. Omalizumab reverses the phenotypic and functional effects of IgE-enhanced Fc epsilonRI on human skin mast cells. J. Immunol. 179, 1353-1361 (2007).

13. MacGlashan, D. W. Jr, Peters, S. P., Warner, J. \& Lichtenstein, L. M Characteristics of human basophil sulfidopeptide leukotriene release: releasability defined as the ability of the basophil to respond to dimeric crosslinks. J. Immunol. 136, 2231-2239 (1986).

14. Hamilton, R. G., Marcotte, G. V. \& Saini, S. S. Immunological methods for quantifying free and total serum IgE levels in allergy patients receiving omalizumab (Xolair) therapy. J. Immunol. Methods 303, 81-91 (2005).

15. Braren, I. et al. Quantitation of serum IgE by using chimeras of human IgE receptor and avian immunoglobulin domains. Anal. Biochem. 412, 134-140 (2011).

16. Magerl, M. et al. Effective treatment of therapy-resistant chronic spontaneous urticaria with omalizumab. J. Allergy Clin. Immunol. 126, 665-666 (2010).

17. Maurer, M. et al.Omalizumab for the treatment of chronic idiopathic or spontaneous urticaria. N. Engl. J. Med. 368, 924-935 (2013).

18. Drinkwater, N. et al. Human immunoglobulin E flexes between acutely bent and extended conformations. Nat. Struct. Mol. Biol. 21, 397-404 (2014).

19. Garman, S. C., Wurzburg, B. A., Tarchevskaya, S. S., Kinet, J. P. \& Jardetzky, T. S. Structure of the $\mathrm{Fc}$ fragment of human IgE bound to its high-affinity receptor Fc epsilonRI alpha. Nature 406, 259-266 (2000).

20. Wurzburg, B. A. \& Jardetzky, T. S. Conformational flexibility in immunoglobulin E-Fc 3-4 revealed in multiple crystal forms. J. Mol. Biol. 393, 176-190 (2009).

21. Holdom, M. D. et al. Conformational changes in IgE contribute to its uniquely slow dissociation rate from receptor FcvarepsilonRI. Nat. Struct. Mol. Biol. 18 571-576 (2011)

22. Dhaliwal, B. et al. Crystal structure of IgE bound to its B-cell receptor CD23 reveals a mechanism of reciprocal allosteric inhibition with high affinity receptor FcepsilonRI. Proc. Natl Acad. Sci. USA 109, 12686-12691 (2012).

23. Pennington, L. F. et al. Structural basis of omalizumab therapy and omalizumab-mediated IgE exchange. Nat. Commun. 7, 11610, (2016)

24. Hamers-Casterman, C. et al. Naturally occurring antibodies devoid of light chains. Nature 363, 446-448 (1993).

25. Ward, E. S., Gussow, D., Griffiths, A. D., Jones, P. T. \& Winter, G. Binding activities of a repertoire of single immunoglobulin variable domains secreted from Escherichia coli. Nature 341, 544-546 (1989).

26. WO2012'/175740A1. Immunoglobulin single variable domains directed against IgE. WO 2012'/175740 A1 (2012).

27. Shade, K. T. et al. A single glycan on IgE is indispensable for initiation of anaphylaxis. J. Exp. Med. 212, 457-467 (2015).

28. Plomp, R. et al. Site-specific $\mathrm{N}$-glycosylation analysis of human immunoglobulin E. J. Proteome Res. 13, 536-546 (2014).

29 . Wan, T. et al. The crystal structure of IgE Fc reveals an asymmetrically bent conformation. Nat. Immunol. 3, 681-686 (2002).

30. Cohen, E. S. et al. A novel IgE-neutralizing antibody for the treatment of severe uncontrolled asthma. $m A b s$ 6, 756-764 (2014).

31. Kim, B. et al. Accelerated disassembly of IgE-receptor complexes by a disruptive macromolecular inhibitor. Nature 491, 613-617 (2012).

32. Shamji, M. H. et al. Cell-free detection of allergen-IgE cross-linking with immobilized phase CD23: inhibition by blocking antibody responses after immunotherapy. J. Allergy Clin. Immunol. 132, 1003-1005 e1001-1004 (2013).

33. Tria, G., Mertens, H. D., Kachala, M. \& Svergun, D. I. Advanced ensemble modelling of flexible macromolecules using X-ray solution scattering. IUCrJ 2, 207-217, (2015).

34. Selb, R. et al. Critical and direct involvement of the CD23 stalk region in IgE binding. J Allergy Clin Immunol 139, 281-289.e5 (2017).

35. Clement, M. J. et al. Toward a better understanding of the basis of the molecular mimicry of polysaccharide antigens by peptides: the example of Shigella flexneri 5a. J. Biol. Chem. 281, 2317-2332 (2006).

36. Wilcock, L. K., Francis, J. N. \& Durham, S. R. IgE-facilitated antigen presentation: role in allergy and the influence of allergen immunotherapy Immunol. Allergy Clin. North. Am. 26, 333-347 (2006).

37. van Neerven, R. J. et al. Humanized anti-IgE mAb Hu-901 prevents the activation of allergen-specific T cells. Int. Arch. Allergy Immunol. 124, 400-402 (2001).

38. Klunker, S. et al. Combination treatment with omalizumab and rush immunotherapy for ragweed-induced allergic rhinitis: inhibition of IgEfacilitated allergen binding. J. Allergy Clin. Immunol. 120, 688-695 (2007).

39. Christensen, L. H., Holm, J., Lund, G., Riise, E. \& Lund, K. Several distinct properties of the IgE repertoire determine effector cell degranulation in response to allergen challenge. J. Allergy Clin. Immunol. 122, 298-304 (2008). 
40. Eggel, A. et al. Accelerated dissociation of IgE-FcepsilonRI complexes by disruptive inhibitors actively desensitizes allergic effector cells. J. Allergy Clin. Immunol. 133, 1709-1719 (2014).

41. Macglashan, D. W. Jr \& Saini, S. S. Omalizumab increases the intrinsic sensitivity of human basophils to IgE-mediated stimulation. J. Allergy Clin. Immunol. 132, 906-911 (2013).

42. Konning, D. et al. Camelid and shark single domain antibodies: structural features and therapeutic potential. Curr. Opin. Struct. Biol. 45, 10-16 (2016).

43. Detalle, L. et al. Generation and characterization of ALX-0171, a potent novel therapeutic nanobody for the treatment of respiratory syncytial virus infection. Antimicrob. Agents Chemother. 60, 6-13 (2015).

44. Fahy, J. V. et al. Effect of aerosolized anti-IgE (E25) on airway responses to inhaled allergen in asthmatic subjects. Am. J. Respir. Crit. Care. Med. 160, 1023-1027 (1999).

45. Evans, G., Alianelli, L., Burt, M., Wagner, A. \& Sawhney, K. J. S. in AIP Conference Proceedings on Diamond Beamline 124: A Flexible Instrument for Macromolecular Micro-Crystallography 836 (2007).

46. Kabsch, W. XDS. Acta Crystallogr. D Biol. Crystallogr. 66, 125-132 (2010).

47. McCoy, A. J. et al. Phaser crystallographic software. J. Appl. Crystallogr. 40, 658-674 (2007).

48. Emsley, P. \& Cowtan, K. Coot: model-building tools for molecular graphics. Acta Crystallogr. D Biol. Crystallogr. 60, 2126-2132 (2004).

49. Adams, P. D. et al. PHENIX: a comprehensive Python-based system for macromolecular structure solution. Acta Crystallogr. D Biol. Crystallogr. 66, 213-221 (2010).

50. Croll, T. I. \& Andersen, G. R. Re-evaluation of low-resolution crystal structures via interactive molecular-dynamics flexible fitting (iMDFF): a case study in complement C4. Acta crystallographica Sect. D. Struct. Biol. 72, 1006-1016 (2016).

51. Braren, I. et al. Generation of human monoclonal allergen-specific IgE and IgG antibodies from synthetic antibody libraries. Clin. Chem. 53, 837-844 (2007).

52. Johansson, S. G. et al. Passive IgE-sensitization by blood transfusion. Allergy $\mathbf{6 0}$, 1192-1199 (2005).

53. Hecker, J. et al. Generation and epitope analysis of human monoclonal antibody isotypes with specificity for the Timothy grass major allergen Phl p 5a. Mol. Immunol. 48, 1236-1244 (2011).

54. Mobs, C. et al. Decline of Ves v 5-specific blocking capacity in wasp venom-allergic patients after stopping allergen immunotherapy. Allergy 70, 715-719 (2015)

55. Pernot, P. et al. Upgraded ESRF BM29 beamline for SAXS on macromolecules in solution. J. Synchrotron Radiat. 20, 660-664 (2013).

56. Brennich, M. E. et al. Online data analysis at the ESRF bioSAXS beamline, BM29. J. Appl. Crystallogr. 49, 203-212 (2016).

57. Franke, D., Kikhney, A. G. \& Svergun, D. I. Automated acquisition and analysis of small angle X-ray scattering data. Nucl. Instrum. Meth A 689, 52-59 (2012).

58. Svergun, D. I. Determination of the regularization parameter in indirect-transform methods using perceptual criteria. J. Appl. Crystallogr. 25, 495-503 (1992).

59. Petoukhov, M. V. et al. New developments in the ATSAS program package for small-angle scattering data analysis. J. Appl. Crystallogr. 45, 342-350 (2012).
60. Hayward, S. \& Berendsen, H. J. Systematic analysis of domain motions in proteins from conformational change: new results on citrate synthase and T4 lysozyme. Proteins 30, 144-154 (1998).

\section{Acknowledgements}

We acknowledge the help of Jesper Lykkegaard Karlsen with refinement and Manuel Schulze-Dasbeck for expert technical assistance. We are grateful to local contact at the ESRF for providing assistance in using beamline BM29. The authors would like to thank Diamond Light Source for beamtime, and the staff of beamlines I02, I03, and I24 for assistance with crystal testing and data collection. This work was supported in part by a research grant of the Danish Council for Independent Research to E.S. G.R.A. was supported by the Danish Council for Independent Research, the Lundbeck foundation, and Danscatt, N.S.L. was supported by the Lundbeck Foundation.

\section{Author contributions}

The study was conceived by F.J., M.P., N.S.L., R.K.J., C.M., G.R.A., and E.S. F.J., M.P., N.S.L., R.K.J., B.M., M.M., and Ma.Ma. participated in all stages of the project and performed the experiments. G.R.A., N.S.L., and E.S. wrote the manuscript together with F.J., M.P., N.S.L., C.M., T.J., and R.K.J. C.M., M.M.R., and W.P. provided and analyzed the patient material. All authors contributed to the interpretation of the data and provided critical review of the manuscript.

\section{Additional information}

Supplementary Information accompanies this paper at https://doi.org/10.1038/s41467017-02312-7.

Competing interests: The authors declare no competing financial interests.

Reprints and permission information is available online at http://npg.nature.com/ reprintsandpermissions/

Publisher's note: Springer Nature remains neutral with regard to jurisdictional claims in published maps and institutional affiliations.

\begin{abstract}
cc (i) Open Access This article is licensed under a Creative Commons Attribution 4.0 International License, which permits use, sharing, adaptation, distribution and reproduction in any medium or format, as long as you give appropriate credit to the original author(s) and the source, provide a link to the Creative Commons license, and indicate if changes were made. The images or other third party material in this article are included in the article's Creative Commons license, unless indicated otherwise in a credit line to the material. If material is not included in the article's Creative Commons license and your intended use is not permitted by statutory regulation or exceeds the permitted use, you will need to obtain permission directly from the copyright holder. To view a copy of this license, visit http://creativecommons.org/ licenses/by/4.0/.
\end{abstract}

(C) The Author(s) 2017 